\title{
LAS TIC EN EL AULA MULTIGRADO. UNA EXPERIENCIA DE FORMACIÓN DE PROFESORES
}

\section{ICT IN THE MULTIGRADE CLASSROOM. A TEACHER TRAINING EXPERIENCE}

\author{
Yaneth Cucunubá Tabaco \\ Nohora Elisabeth Alfonso Bernal \\ Carmen Helena Cepeda Araque \\ Universidad Pedagógica y Tecnológica de Colombia
}

\begin{abstract}
RESUMEN
El propósito de la investigación fue implementar un programa de formación que permitiera fortalecer las competencias TIC de los profesores con aula multigrado, a través de la metodología de Investigación Acción Educativa, fundamentada en la reflexión, reconstrucción y valoración de la práctica pedagógica de los
\end{abstract}

profesores. Dentro de los resultados obtenidos se evidencia que los profesores comprenden la importancia de las TIC y se interesan por implementarlas en sus prácticas pedagógicas, que la formación de profesores en competencias TIC debe propender el uso pedagógico de las mismas y que la conectividad a internet es

1 Investigadora Grupo de Investigación para la Animación Cultural Muisuata, Licenciada en Informática Educativa, estudiante de Maestría en TIC Aplicadas a las Ciencias de la Educación, Universidad Pedagógica y Tecnológica de Colombia, Duitama, Colombia. E-mail: yaneth.cucunuba@uptc.edu.co ORCID ID: https://orcid.org/0000-0003-4720-1718

2 Investigadora y directora Grupo de Investigación para la Animación Cultural Muisuata, Doctora en Ciencias de la Educación, Magíster en Desarrollo Educativo y Social, Especialista en Recreación Educativa, Profesional en Administración Turística y Hotelera. Universidad Pedagógica y Tecnológica de Colombia, Duitama, Colombia. E-mail: nohora.alfonso@uptc.edu.co ORCID ID: https://orcid.org/0000-0002-7831-7433

3 Investigadora Grupo de investigación GIE, Magíster en Estadística, Especialista en Pedagogía para el Desarrollo del Aprendizaje Autónomo, Especialista en Matemáticas y Estadística Aplicada, Licenciada en Matemáticas y Física. Universidad Pedagógica y Tecnológica de Colombia, Duitama, Colombia. E-mail: carmen.cepeda@uptc.edu.co ORCID ID https://orcid. org/0000-0002-1317-0776 
fundamental pero no debe ser una limitante para implementar las TIC en el proceso de enseñanza y aprendizaje.

\section{PALABRAS CLAVE}

Formación de profesores, educación rural, aula multigrado, competencias TIC, prácticas pedagógicas

\section{ABSTRACT}

The purpose of the research was to implement a training program that would strengthen the ICT competences of teachers with a multi-grade classroom, through the Educational Action Research methodology, based on the reflection, reconstruction and evaluation of teachers' pedagogical practice. Within the results obtained, it is evident that teachers understand the importance of ICT and are interested in implementing it in their pedagogical practices, that the training of teachers in ICT competences should promote the pedagogical use of ICTs and that internet connectivity is essential but it should not be a limitation to implement ICT in the teaching and learning process

\section{KEY WORDS}

Teacher training, rural education, multigrade classroom, ICT skills, pedagogical practices

\section{INTRODUCCIÓN}

En Colombia como en muchos países del mundo la preocupación por mejorar la calidad de la educación es cada vez más visible, los gobiernos han propuesto políticas educativas que buscan la integración de diferentes aspectos que permitan mejorar los procesos de enseñanza y aprendizaje, una de las estrategias es la implementación de las Tecnologías de la Información y la Comunicación -TIC- en el aula, la idea de integrar las TIC es lograr que el educando por medio de las tecnologías logre desarrollar diversas competencias y apropiar de forma fácil y significativa el conocimiento. Para lograr esa integración se requiere que los profesores apropien las competencias TIC y las integren a los procesos de enseñanza, como un aporte para:

comprender con mayor profundidad y conciencia crítica el tema de las prácticas investigativas, en tanto no solo inciden en la cualificación de la investigación educativa, sino que afecta la democratización de la vida social, como posibilidad de ampliar el acceso de oportunidades para lograr mayor autonomía individual y colectiva desde el quehacer sistemático, riguroso, crítico y propositivo de una condición netamente humana: la producción de conocimiento. Alfonso (2015, p. 73 )

Este artículo muestra la importancia de la formación de profesores en competencias TIC para el aula multigrado en la escuela rural. Tiene en cuenta tres tópicos importantes: la educación rural que históricamente ha sido considerada y proyectada bajo los mismos parámetros de la educación urbana, dejando de lado las características propias de su entorno; el aula multigrado que es la característica principal de la educación rural y se identifica por tener varios grados en el aula, orientados por un solo profesor o profesora, así mismo presentan edades diferentes $\mathrm{y}$, la formación de profesores en competencias TIC que en los últimos años ha tomado gran importancia desde el ámbito nacional e internacional, no obstante muchas escuelas del sector rural que no cuentan con servicio de internet tienden a ser excluidas de estos proyectos de formación de profesores.

En Colombia, la formación de profesores en competencias TIC se ha venido desarrollando con algunos procesos liderados y ejecutados por computadores para educar, sin embargo, es importante tener en cuenta que muchas 
de las escuelas rurales no cuentan con los recursos tecnológicos, están ubicadas en lugares sin o con escasa cobertura telefónica y la conectividad a internet es limitada o nula, por lo que se debe enfatizar en las TIC offline, de esta forma la conectividad no sería una limitante para el profesor en el momento de aplicar las TIC como mediación en el aula de clases.

En el informe Sobre Tendencias Sociales y Educativas en América Latina. Realizado por López, Lugo \& Toranzos (2014) hacen una exposición respecto a los desafíos que se presentan en los sistemas educativos de América Latina, referente a varios aspectos que influyen en la implementación de las TIC en el aula, dentro de los cuales resaltan las políticas de integración de las TIC en los sistemas educativos, las diferencias generacionales, las principales tendencias que asumen las políticas TIC, la situación de infraestructura, equipamiento y conectividad en los diferentes países de América Latina, la inclusión digital, la integración de las TIC a las instituciones educativas y la formación inicial y de desarrollo profesional de profesores. En este informe los autores exponen que:

En América Latina, la brecha digital resulta notable no solo cuando se realiza la comparación con los países integrantes de la OCDE, sino en las desigualdades que exhiben los países internamente. Sobre todo, a nivel territorial (entre la población urbana y la rural) [...] y en los países en los que se cuenta con datos, resulta evidente el rezago existente en el caso de las zonas rurales. Esto implica que la disponibilidad de Internet en los hogares rurales puede ser 5 a 10 veces menor que en los hogares urbanos (López, et al., 2014, p.77)
Para el caso de Colombia De Zubiría, (2020). En su artículo titulado La educación en tiempos de cuarentena, expone las dificultades que se presentan en materia de cobertura de equipamientos, conectividad a internet y acceso a la información, mostrando que "en Colombia solo el $4 \%$ de los municipios tiene buena conectividad, el $63 \%$ de los bachilleres del 2018 no tenía acceso a Internet desde sus hogares y, en las zonas rurales, tan solo el $9 \%$ de los jóvenes disponen de computador". De igual manera hace énfasis en la cualificación de los profesores en competencias digitales de tal forma que se puedan utilizar los distintos dispositivos dándole un uso pedagógico para fortalecer la creatividad, la autonomía, la solidaridad y la lectura; continúa Zubiria (2020) "Los docentes tienen la responsabilidad de cualificar su formación pedagógica y digital, el MEN la responsabilidad de brindar las condiciones para que sea posible y entre todos, tenemos que garantizar la calidad de la educación de la cual hemos carecido".

Esta investigación muestra la información obtenida en los momentos del desarrollo de la metodología de investigación, de la siguiente forma: la reflexión de la práctica pedagógica con los profesores, la deconstrucción de la práctica y la valoración de la práctica pedagógica reconstruida. Para reflexionar la práctica pedagógica con los profesores, inicialmente se hizo una revisión teórica sobre prácticas pedagógicas, luego de forma colectiva con los profesores participantes en la investigación se hace la reflexión de las practicas pedagógicas mediadas por las TIC, esta reflexión se basa en cuatro (4) categorías a saber: planeación y preparación de clases, ambientes para el aprendizaje, practica pedagógica y responsabilidades profesionales, fundamentado en el marco profesoral de Danielson (2013), de igual manera se tomó como referencia el documento Competencias TIC para el desarrollo profesional docente, expedido por el Ministerio de Educación Nacional- MEN- en el año 
2013. Un segundo momento, donde se hace la reconstrucción de la práctica pedagógica teniendo en cuenta los resultados obtenidos en el primer momento y se consensa con los profesores la planeación para el proceso de formación y en el tercer momento donde se hace la valoración de la práctica pedagógica reconstruida para ver la efectividad del proceso de formación. Por último, se relacionan algunos criterios que fortalecen un proceso de formación de profesores en TIC, estos criterios surgen a partir del análisis de la información obtenida en los estudios realizados por los autores referenciados, lo observado en el proceso de formación y las opiniones de los profesores participantes.

\section{REFERENTES TEÓRICOS}

\subsection{Formación de Profesores en} Competencias TIC, Educación Rural y aula multigrado

Respecto a la formación de profesores en competencias TIC, educación rural y aula multigrado, se tomaron como referente a nivel internacional cinco (5) documentos: 3 trabajos de investigación y 2 Informes de la UNESCO. Y, en el ámbito nacional se tomaron siete (7) estudios sobre la temática, A nivel internacional se retoma a Sobrino, Sastre, Arranz, Lema \& Abellán (2013), en su trabajo "Oportunidades y desafíos en el desarrollo profesional de docentes con TIC en una escuela rural en España: una mirada a la Escuela Nueva", los autores hacen una reflexión sobre los retos y oportunidades que presentan las TIC a los profesores en su crecimiento profesional. El estudio es desarrollado en tres apartados: primero muestran algunos acontecimientos históricos y su impacto en la Escuela Nueva en España, luego retoman un estudio de caso sobre formación de profesores desarrollado en una escuela rural por un periodo de tres años, este pretendía buscar evidencias sobre "cómo promover la creación y compartición de diseños educativos innovadores en el uso de las TIC a través de una plataforma tecnológica consensuada y compartida por una comunidad de aprendizaje" (Sobrino, et al., 2013, p. 185) y por último presentan evidencias sobre los efectos que asume la escuela nueva frente al desarrollo de competencias profesionales de los profesores en el siglo XXI.

De la misma forma, Almenara y Diaz (2014) en su estudio "Miradas sobre la formación del profesorado en Tecnologías de Información y Comunicación (TIC)", presentan un análisis e informe detallado de los aspectos teóricos - conceptuales respecto a la formación de profesores en TIC, fundamentan su desarrollo en la investigación descriptiva documental, por medio de la cual los autores obtuvieron bases necesarias para dar una visión innovadora respecto a la propuesta de formación de profesores y las necesidades que se deben consideran teniendo en cuenta los diferentes contextos. logrando concluir que:

los profesores necesitan saber cómo la tecnología puede y debe ser usada por los estudiantes para mejorar su propio aprendizaje, esto conlleva a la necesidad de un profesor, no tan formado para el manejo instrumental de las tecnologías, sino más bien para el posible aprovechamiento educativo que los alumnos pueden hacer de estas. (Almenara y Diaz, 2014, p. 20)

Lugo (2010), en su estudio "Las políticas TIC en la educación de América Latina. Tendencias y experiencias" expone que las políticas educativas en américa latina tienen presente el uso de las TIC como recurso fundamental en los procesos educativos, pero la realidad de pobreza y desigualdad social repercuten en el ámbito educativo. En este sentido, América Latina presenta grandes desafíos como "universalizar la educación básica y media, incorporar los 
sectores sociales excluidos, mejorar la calidad y masificar la enseñanza superior y afrontar la deserción y repitencia" (Lugo,2010, p.25). Y continua Lugo "las TIC no son la panacea para los problemas educativos sino una ventana de oportunidad para innovar en la gestión del conocimiento y en las estrategias de enseñanza" (2010, p.52).

De igual manera, Instituciones como Organización de las Naciones Unidas para la Educación, la Ciencia y la Cultura (UNESCO), en su Informe sobre Aplicación de las TIC para el acceso a una educación de calidad para la población rural (2007), expone que, debido a las características que presenta la educción rural en relación al contexto urbano frente a los índices de pobreza, aislamiento, falta de recursos básicos:

las TIC constituyen una oportunidad para mejorar la calidad de la educación en el medio rural, en cuanto que pueden hacer más fácil el acceso de las poblaciones a una educación relevante y pertinente para ellas. Sin embargo, el uso de las TIC plantea también el desafío de lograr una mayor equidad, pues se corre el riesgo de que se agrande la brecha entre quienes tienen acceso a ellas y quienes no lo tienen. (UNESCO, 2007, p. 3)

De igual forma, la UNESCO revela que el primer indicio de la integración de las TIC a la educación viene desde la educación "por radio en la década de los años 20 y la enseñanza asistida por televisión desde los años 50. Estas formas de enseñanza asistida por TIC, actualmente consideradas antiguas, han demostrado ser eficientes a gran escala y de bajo costo" (UNESCO, 2013, p. 11). Es por esto que, en estas épocas con la pandemia presentada por el Covid-19, estas dos formas de implementación de las TIC han tomado gran relevancia en entornos rurales y urbanos donde no se cuenta con los recursos necesarios de conectividad a internet.

A nivel de Colombia se revisaron aproximadamente 24 estudios realizados sobre educación rural y formación de profesores en TIC para el aula multigrado, las temáticas abordadas en estos trabajos son: educación rural, formación de profesores, prácticas pedagógicas, políticas educativas, las TIC en escuelas rurales, las TIC en el modelo Escuela Nueva y experiencias de los docentes en la implementación de las TIC en las escuelas multigrado.

Las investigaciones que directamente se relacionan con la problemática de formación de profesores y la integración de las TIC en la escuela rural con aula multigrado son las que se referencian a continuación: Álvarez y Romero (2015) presentan un trabajo titulado "Percepciones de los docentes rurales sobre las TIC en sus prácticas pedagógicas," el estudio fue desarrollado con profesores de la escuela rural pertenecientes al departamento de Boyacá y Cundinamarca y pretende exponer las percepciones que ellos tienen sobre la implementación de las TIC en sus prácticas pedagógicas teniendo en cuenta las características propias del contexto rural. En este sentido, el estudio realizado pudo concluir que: "es muy importante partir de las experiencias y percepciones los docentes rurales, para hacer una buena apropiación del TIC en los procesos pedagógicos" (Alvarez y Romero, 2015, p. 391).

Hernández, Jurado y Romero (2014) en un estudio titulado "Análisis de publicaciones hispanoamericanas sobre TIC en escuelas y zonas rurales", el estudio muestra los resultados de una revisión de publicaciones sobre la implementación de las TIC en escuelas rurales, dentro de los aspectos relevantes obtenidos en el análisis se evidencia: 
la preocupación por el estado actual de la escuela rural, la instrumentalización de las TIC y el escaso apoyo a sus docentes. Se encontraron avances conceptuales prometedores provenientes de distintas disciplinas. La transformación socio-educativa se condiciona a las políticas públicas sectoriales y recomiendan adoptar una postura cultural para comprender la cotidianidad de las TIC en las escuelas rurales. (Hernández, et ál., 2014, p. 103)

Uribe (2015) desarrolla un proyecto titulado "Diseño de una propuesta curricular para docentes de la básica primaria, enfocada a la integración de las Tecnologías de la Información y las Comunicaciones (TIC) en el modelo escuela nueva - escuela activa, en la institución educativa rural (IER) Santa Rosa de Lima del Municipio de Giraldo" la finalidad del estudio desarrollado fue fortalecer las competencias TIC del profesorado mediante la elaboración de material educativo digital, de tal forma que contribuya a la transformación de las prácticas pedagógicas de los profesores y dinamice los procesos de enseñanza aprendizaje desde luego manteniendo las características propias del Modelo Escuela Nueva.

Rodríguez y Saavedra (2018) en su trabajo "Las TIC: Una oportunidad para fortalecer procesos de enseñanza en el marco del modelo escuela nueva" desarrollan un análisis reflexivo respecto a la integración de las TIC en las prácticas pedagógicas de los profesores con modelo escuela nueva, para su desarrollo se hace una contextualización del entorno y se revisan experiencias a nivel nacional e internacional que sustenten su importancia. A partir de la investigación los autores concluyen "que es necesario fortalecer las competencias TIC de los educadores en coherencia con el enfoque de la escuela multigrado para favorecer procesos de aprendizaje en los estudiantes" (Rodríguez y Saavedra, 2018, p. 39). De igual manera es importante generar cambios significativos en los procesos académicos mediados por las TIC de tal forma que fortalezcan las competencias digitales en las comunidades educativas rurales.

Carreño, de la Garza \& Zermeño (2016) en su estudio "Experiencias de los docentes en la implementación de las TIC en escuelas rurales multigrado", el estudio fue desarrollado en tres escuelas con aula multigrado correspondientes al sector rural en una misma institución educativa, se reflexiona sobre la implementación de las TIC en las prácticas de aula. El análisis de la información obtenida arroja como resultados:

una percepción positiva de las maestras y estudiantes respecto a la presencia de los computadores en el aula de clases, estos se perciben como un elemento agradable, motivador y facilitador de todo tipo de actividades y se concluye que la inclusión de los computadores en la cotidianidad de las escuelas rurales permite entornos más dinámicos y motivadores, facilita el manejo de cursos multigrado por parte del docente y promueve el trabajo colaborativo. (Carreño, et ál., 2016, p. 53)

De igual forma se presenta un trabajo desarrollado por Herrera y Buitrago (2015) "Educación rural en Boyacá, fortalezas y debilidades desde la perspectiva del profesorado" Este trabajo fue efectuado en las provincias de Centro, Tundama y Sugamuxi del departamento de Boyacá y muestra una relación entre las fortalezas y debilidades que se presentan en la educación desde el ámbito rural y urbano, teniendo en cuenta algunos aspectos relacionados con el contexto escolar y las percepciones de los profesores, dentro de los resultados obtenidos se evidencia que las condiciones de cada institución varía de acuerdo a los profesores asignados, el número de estudiantes y los 
servicios reglamentarios con los cuales cuente la institución.

De otra parte, un estudio realizado por Molina y Mesa (2018) "Las TIC en escuelas rurales: realidades y proyección para la integración", presenta un análisis sobre el efecto de las políticas gubernamentales respecto a la implementación de las TIC en el aula, resaltando aspectos importantes relacionados con infraestructura computacional, uso de los recursos por parte de los profesores, y las competencias de los profesores para implementar las TIC en los procesos de enseñanza y aprendizaje. En este respecto la investigación permitió revelar las necesidades y preocupaciones de los profesores. De igual manera, a partir de sus opiniones y experiencias se generó "una secuencia para la incorporación de las TIC para los centros educativos rurales, que incluye la planificación, capacitación de maestros, disposición y gestión de recursos, mantenimiento y seguimiento e incentivos" (Molina y Mesa, 2018, p. 75).

\section{METODOLOGÍA}

El enfoque que privilegia esta investigación es cualitativo, ya que permite participar minuciosamente en el estudio y análisis de las actividades propuestas para obtener información sobre la situación o problema que se vaya a investigar, de igual manera, facilita la interacción con la población participante. Para este caso en particular las prácticas pedagógicas mediadas por las TIC, de los profesores de la I. E las Mercedes con aula multigrado.

Rodríguez(2011), manifiestaque“Lainvestigación cualitativa esencialmente desarrolla procesos en términos descriptivos e interpreta acciones, lenguajes, hechos funcionalmente relevantes y los sitúa en una correlación con el más amplio contexto social" (p.10). De esta manera en el proceso se desarrollaron diversas actividades que permitieron registrar las opiniones de los profesores respecto a la reflexión sobre la implementación de las TIC en el aula, de igual forma las propuestas de cada uno de ellos para mejorar los procesos de enseñanza teniendo en cuenta el contexto y la población estudiantil. Todo este proceso se hizo tomando en cuenta las perspectivas de los participantes.

El tipo de investigación en el cual se basó el desarrollo de este estudio es Investigación Acción Educativa-IAE-, entendida por Restrepo (2002) como aquella que puede. "enfocarse a transformar Instituciones escolares totales 0 unidades o prácticas sociales de la misma, o puede circunscribirse a la práctica pedagógica de un docente particular" (p.4) de igual forma Restrepo (2002) plantea tres momentos para el desarrollo de la investigación acción educativa: la reflexión o deconstrucción de la práctica, la reconstrucción de la práctica y la validación de la práctica alternativa o reconstruida. Con base en la metodología planteada por Restrepo en este proyecto se desarrollaron tres momentos de la siguiente forma:

Figura 1. Estructura de la metodología

La población objeto de investigación la constituye un grupo de profesores de la Institución Educativa las Mercedes de la vereda el Caucho en el municipio de Nunchía en el departamento de Casanare, se trabajó con los profesores de aula multigrado de la sede central y de las sedes Cazadero y Rojas Pinilla. 


\section{RESULTADOS}

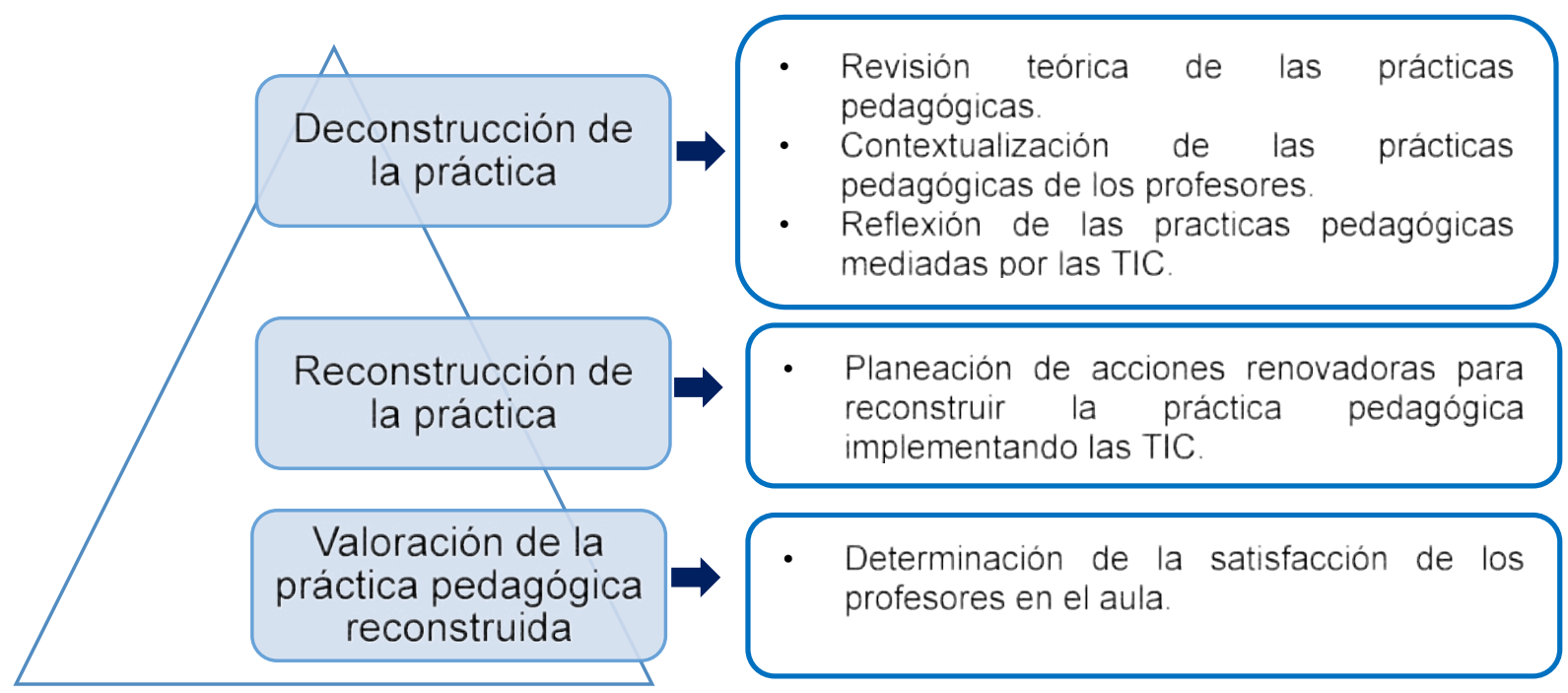

Fuente: Cucunubá (2020)

Se hace una presentación de los resultados obtenidos en los tres momentos de la Investigación Acción Educativa, haciendo énfasis en la valoración de la práctica pedagógica. Para la reflexión de la práctica pedagógica se tomó como referencia los componentes planteados por Danielson (2013) en el Marco Profesoral para la Enseñanza, y se hizo una autorreflexión con los profesores respecto a la práctica pedagógica que desarrollan a diario con sus estudiantes. El análisis se hizo con base a cuatro (4) enfoques que se relacionan a continuación: planeación de clases, ambientes para el aprendizaje, práctica pedagógica y responsabilidades profesionales.

\subsection{Reflexionando la práctica pedagógica}

En este apartado se desarrolló el análisis y reflexión de la práctica pedagógica mediada por las TIC de los profesores de la I.E. las Mercedes con aula multigrado, de este proceso se obtuvieron algunos resultados que se relacionan a continuación: En cuanto a la planeación de clases, se evidencio que los profesores implementan diversas estrategias para la enseñanza, conocen el contexto de sus estudiantes, promueven el respeto por los acuerdos de aula y las normas para la convivencia y fomentan el diálogo continuo con estudiantes, padres o cuidadores, pero en cuanto a las TIC, están no están articuladas en el PEI, no se ven en la planeación y solo dos profesores las integran de forma incipiente por medio de videos, presentaciones y texto.

Respecto a los ambientes para el aprendizaje, manejan un ambiente acorde a la actividad que se esté desarrollando, tienen en cuenta los ritmos y estilos de aprendizaje de sus estudiantes y promueven la importancia de un clima agradable, respetuoso, participativo y armonioso, generando acuerdos de aula para fortalecer la sana convivencia. Así mismo se evidencia que se debe fortalecer el trabajo colaborativo y cooperativo para aprovechar las capacidades y destrezas de los estudiantes.

Referenteala práctica pedagógica, los profesores manifiestan que es un reto la implementación de las TIC en el aula multigrado y para lograrlo se debe ser muy organizado en la planeación, tener las competencias TIC necesarias que faciliten el acceso a diferentes recursos que se puedan implementar offline debido a la falta de conectividad, de igual forma manifiestan la importancia de integrar las TIC en todas las 
asignaturas de tal forma que el aprendizaje sea más dinámico y facilite el desarrollo de actividades innovadoras, promoviendo en los estudiantes el trabajo autónomo, cooperativo y colorativo enriqueciendo así su conocimiento. Igualmente, la integración de las TIC enriquece el saber pedagógico del profesor y fortalece sus competencias digitales.

Por último, las responsabilidades profesionales. Se evidencio que el $75 \%$ de los profesores participantes no desarrollan procesos de profesionalización por medio de las TIC y la totalidad de ellos no participan en comunidades de aprendizaje; pero están conscientes que la implementación de las TIC en el aula es importante puesto que permite desarrollar prácticas motivadoras, facilitan la sistematización de la información, la actualización continua, favorece las habilidades en los procesos de enseñanza y promueven la innovación. De igual forma afirman que se debe reflexionar continuamente con los colegas sobre la práctica pedagógica para enriquecer su conocimiento y conocer experiencias de otros compañeros que ayuden a mejorar sus procesos de enseñanza y aprendizaje.

\subsection{Reconstruyendo la práctica pedagógica}

Reconstruir la práctica pedagógica implica hacer un análisis del desarrollo del quehacer diario en el aula, teniendo en cuenta diferentes aspectos como la interacción reciproca profesor estudiante, la comunicación, los ambientes para el aprendizaje, los ritmos de aprendizaje de los estudiantes, las concepciones culturales, entre otros factores sobre los cuales se debe reflexionar para fortalecer y mejorar la práctica pedagógica y a partir de ese análisis comenzar a innovar para mejorar los procesos de enseñanza y aprendizaje. Quero (2006) manifiesta que "EI docente desde el deber ser de su actuación profesional, como mediador y formador, debe reflexionar sobre su práctica pedagógica para mejorarla y/o fortalecerla y desde esa instancia elaborar nuevos conocimientos" (p.3).

Para hacer la reconstrucción de la práctica pedagógica de los profesores de la I.E. las Mercedes, se tomaron como referente los resultados obtenidos en la reflexión colectiva de las prácticas pedagógicas, a partir de la reflexión se evidenciaron fortalezas y aspectos por cualificar, dentro de las fortalezas se puede resaltar que los profesores tienen un dominio disciplinar y didáctico en el desarrollo de sus prácticas pedagógicas, conocen el contexto de sus estudiantes, manejan una comunicación asertiva y promueven estrategias para mejorar las relaciones interpersonales.

En cuanto a los aspectos por cualificar se evidenció que los profesores no conocían los estándares de competencias TIC, no había articulación de las TIC en la práctica pedagógica, presentaban dificultad para acceder a recursos TIC offline para integrarlos en el aula y no participan en redes de aprendizaje. A partir de estos resultados los profesores priorizaron para el proceso de formación cuatro (4) aspectos: 1. Conocimiento sobre los estándares de competencias TIC para el desarrollo profesional docente, 2. Formación en paquete de Office y otras aplicaciones offline, 3. Formación en diseño de material educativo digital, y 4. Manejo de buscadores, navegadores y portales educativos en internet. El proceso de formación se desarrolló en base a temáticas propias del plan de estudios, de tal forma que pudiera aplicarse luego en el aula con los estudiantes. Los resultados obtenidos en este proceso se muestran a continuación:

- Estándares de competencias TIC para el desarrollo profesional docente: en este aspecto los profesores analizaron y comprendieron los estándares de competencias TIC para docentes propuestos por la UNESCO y los estándares de 
competencias TIC para el desarrollo profesional docente propuestos por el MEN de Colombia. A partir del análisis y comprensión de los dos documentos se acordó con los profesores trabajar la competencia pedagógica y tecnología en el proceso de formación, las demás competencias fueron abordadas paulatinamente.

- $\quad$ Paquete de office y otras aplicaciones offline: con ayuda de estas aplicaciones los profesores desarrollaron temáticas propias del plan de estudios de cada asignatura, trabajando de acuerdo al tema o área. En el caso de matemáticas llevaron a cabo actividades aplicando Excel facilitando el fortalecimiento de competencias correspondientes al pensamiento aleatorio y sistemas de datos estipulados en los estándares básicos de competencias, de igual manera desarrollaron actividades propias del pensamiento espacial y sistemas geométricos haciendo uso de autoformas. En las demás asignaturas desarrollaron actividades en PowerPoint para el diseño y edición de presentaciones, en Word para el manejo y edición de información, la organización de ideas de forma gráfica en Cmaptools y editores de dibujo para los niños más pequeños.

- $\quad$ Diseño de material educativo digital: el diseño de material educativo digital se desarrolló en Edilim y se enfocó a una temática en particular obtenida del plan de estudios, este diseño permitió a los profesores integrar diferentes recursos y aplicaciones para hacerlo llamativo e interactivo, al finalizar fue un recurso que se compartió para que cada profesor lo aplicara en su práctica pedagógica con sus estudiantes.

- $\quad$ Manejo de internet: como resultado en este aspecto se logró que cada profesor hiciera su registro en Colombia Aprende y Eduteka, de esta manera pudieron acceder a los recursos educativos que ofrecen estos portales, de igual manera los profesores desarrollaron competencias para buscar, organizary descargar recursos offline como videos, imágenes, software educativo, presentaciones, documentales, canciones, que pudieron implementar con sus estudiantes para el desarrollo de las clases. De igual forma se exploró el portal educativo Icarito, para acceder a diferentes recursos educativos que facilitan aprender a aprender de forma dinámica.

\subsection{Valorando la práctica reconstruida}

La valoración de la práctica pedagógica reconstruida con los profesores Mercedistas para aula multigrado, se hizo a partir de los resultados obtenidos en la reflexión colectiva con los profesores sobre el proceso de formación, los planeadores de clase, los resultados de la encuesta inicial y final sobre prácticas pedagógicas mediadas por las TIC y el análisis de los registros obtenidos en los diarios de campo llevados por los profesores en el proceso de ejecución de la integración de las TIC en el aula.

Se pudo evidenciar algunos aspectos importantes como: los profesores de la I.E. las Mercedes participantes en el proceso de formación han mejorado sus conocimientos respecto a competencias TIC, en la planeación se observa que proponen actividades para que los estudiantes desarrollen implementando las TIC, de igual manera consultan material educativo en Colombia Aprende y Eduteka, obtienen recursos offline para aplicar en el aula, integran las TIC en diferentes asignaturas, y están seguros que la implementación de las TIC dinamiza los procesos de enseñanza y aprendizaje y fortalece las competencias digitales tanto en los profesores como en los estudiantes.

Dentro de la valoración de la práctica también se identificaron algunas dificultades que evidencian la escasa formación en competencias TIC de los profesores en su formación inicial, por ende, no se tienen las competencias TIC necesarias para integrarlas pedagógicamente lo que ocasiona 
que no se vea la significación de las mismas en el aula por tal razón los dispositivos tecnológicos y recursos TIC terminan siendo subutilizados.

\subsection{Criterios que fortalecen un proceso de formación de profesores en TIC.}

Desde las percepciones de los autores referenciados en esta investigación se puede denotar que la educación rural tiene como principal característica el aula multigrado, de igual forma se presentan una serie de aspectos que hacen de la escuela rural un reto importante para el profesor y aunque "las TIC no son la solución para los problemas educativos si son una oportunidad para innovar en la gestión del conocimiento y en las estrategias de enseñanza" (Lugo, 2010, p.52). esto conlleva a la necesidad de que se logre desarrollar competencias TIC en los profesores para que empiecen a transformar sus prácticas pedagógicas aprovechando los recursos existentes en sus instituciones, fomentando el aprendizaje autónomo, de esta forma, "la inclusión de los computadores en la cotidianidad de los alumnos permite entornos más dinámicos y motivadores, facilita el manejo de cursos multigrado por parte del docente y promueve el trabajo colaborativo" (Carreño, et al.,2016, p.53). en este sentido, es muy importante partir de las experiencias y percepciones de los profesores rurales, para hacer una buena apropiación de las TIC en los procesos pedagógicos.

Fundamentados en la postura de los autores referenciados y los resultados obtenidos en el proceso de formación en competencias TIC desarrollado con los profesores de la I.E. las Mercedes se pudo identificar algunos criterios que son importantes tenerlos en cuenta en el momento de llevar a cabo un proceso de formación de profesores en competencias TIC:

- Tener en cuenta las percepciones y experiencia de los profesores.
- Contar con recursos tecnológicos y apoyo institucional

- Partir de un diagnóstico previo para saber en qué nivel de competencias TIC se encuentran los profesores.

- Nivelar a los profesores que presenten mayor dificultad para apropiar las TIC

- $\quad$ Tener en cuenta ritmos de aprendizaje de los profesores.

- Posibilitar estrategias offline para la integración de las TIC en aula multigrado.

- $\quad$ En el proceso de formación integrar la parte instrumental y pedagógica

- $\quad$ Fortalecer las competencias TIC en relación al modelo de escuela nueva.

- Realimentar y dar continuidad al proceso de formación en competencias TIC

- Orientación de actualización y profesionalización de profesores por medio de las TIC

\section{CONCLUSIONES}

La formación de profesores en competencias TIC debe propender por el uso pedagógico de las mismas, dado que estas dinamizan los procesos de enseñanza y aprendizaje, ayuda al profesor a mejorar de forma significativa sus prácticas pedagógicas, a ser recursivos, creativos e innovadores y en los estudiantes incentiva el gusto por aprender a aprender, desarrolla la creatividad, la toma de decisiones, entre otros; el profesor pasa a ser un orientador del proceso y el estudiante comienza a utilizar las TIC para desarrollar sus propias actividades, integrándolas a su proceso de aprendizaje. 
La cualificación de profesores en competencias TIC debesercorresponsabilidad delosprofesores y el MEN, se debe hacer un proceso continuo de formación y al terminar se debe continuar el apoyo a los profesores ya sea de forma virtual o presencial, Los procesos de formación deben buscar que todos los participantes apropien las competencias, garantizando de esta forma su uso pedagógico.

Implementar las TIC en el aula multigrado no se debe considerar como dificultad, sino como una oportunidad para mejorar, fortalecer habilidades y competencias tanto en el profesor como en el estudiante, ya que los estudiantes de grados superiores pueden apoyar el proceso en los grados iniciales.

La conectividad a internet es una herramienta importante para el proceso de enseñanza y aprendizaje ya que permite la motivación, la búsqueda de información, el acceso a contenidos educativos dinámicos, entre otros; pero no debe ser una limitante para integrar las TIC en el aula, se debe dar relevancia y aprovechar los recursos pedagógicos offline.

\section{REFERENCIAS BIBLIOGRÁFICAS}

Alfonso, N.E. (2015). Representaciones Sociales y Prácticas Investigativas en los Procesos Universitarios en Colombia. En: Calixto, F. R. (Coord.), Representaciones Sociales en la Práctica Educativa y en la Formación Docente (pp.71-86). Toluca, México: Editorial ISCEEM

Almenara, J. C., \& Díaz, V. M. (2014). Miradas sobre la formación del profesorado en tecnologías de información y comunicación (TIC). Enl@ ce: Revista Venezolana de Información, Tecnología y Conocimiento, 11(2), 11-24.

Alvarez-Quiroz, G. B., \& Romero, J. C. B. (2015). Percepciones de los docentes rurales sobre las TIC en sus prácticas pedagógicas. Ciencia, docencia y tecnología, 26(51), 371-394.

Carreño, F. A. F., de la Garza, L. Y. A., \& Zermeño, M. G. G. (2016). Experiencias de los docentes en la implementación de las TIC en escuelas rurales multigrado. EDMETIC, 5(1), 52-72.

Cucunubá, Y. (2020). Las TIC en el aula multigrado. Una propuesta para la formación de profesores [tesis de maestría no publicada]. Universidad Pedagógica y Tecnológica de Colombia. Duitama.

Danielson, C. (2013). A Framework For Teaching. Alexandria, VA: Association for Supervision and Curriculum Development.

De Zubiría Samper. (2020). La educación en tiempos de cuarentena. https:// www.semana.com/opinion/articulo/laeducacion-en-tiempos-de-cuarentenacolumna-de-julian-de-zubiria/661969

Herrera Torres, L., \& Buitrago Bonilla, R. E. (2015). Educación Rural en Boyacá, Fortalezas y debilidades desde la Perspectiva del profesorado. Praxis \& Saber, 6(12), 169-190.

Hernández Sampieri, R., Fernández Collado, C., \& Baptista Lucio, P. (2010). Metodología de la investigación.

Hernández, O., Jurado , H., y Romero, Y. (2014). Análisis de publicaciones hispanoamericanas sobre TIC en escuelas y zonas rurales. Revista Colombiana de Educación. MolinaPacheco, L. E., \& Mesa-Jiménez, F. Y. (2018). Las TIC en escuelas rurales: realidades y proyección para la integración. Praxis \& Saber, 9(21), 75-9. 
Lugo, M. T. (2010). Las políticas TIC en la educación de América Latina. Tendencias y experiencias. Revista Fuentes, 10, 52-68.

Quero, V. D. (2006). Formación docente, práctica pedagógica y saber pedagógico. Laurus, 12(Ext), 88-103.

Restrepo, G. B. (2002). Una variante pedagógica de la investigación-acción educativa. Revista Iberoamericana de educación, 29(1), 1-10.

Restrepo, G. B. (2004). La investigación-acción educativa y la construcción de saber pedagógico. Educación y educadores, (7), 45-56.

Rodríguez, J. M. (2011). métodos de investigación cualitativa qualitative research methods. Revista de la Corporación Internacional para el Desarrollo Educativo BogotáColombia. SILOGISMO, 8.

RODRIGUEZ, S. Y., \& SAAVEDRA, C. E. (2018). Las TIC: Una oportunidad para fortalecer procesos de enseñanza en el marco del modelo escuela nueva. Revista ESPACIOS, 39(49).

Sobrino, S. L. V., Sastre, S. G., Arranz, B. C., Lema, V. G., \& Abellán, I. J. (2013). Oportunidades y desafíos en el desarrollo profesional de docentes con TIC en una escuela rural en España: una mirada a la Escuela Nueva. Revista interuniversitaria de formación del profesorado, 27(3), 185-199.

UNESCO. (2007). Videoconferencia Educación para las poblaciones rurales. Aplicación de las TIC para el acceso a una educación de calidad para la población rural. OREALC.https://unesdoc. unesco.org/ark:/48223/pf0000159966/ PDF/159966spa.pdf.multi
UNESCO. (2013). Uso de TIC en educación en América Latina y el Caribe. Canada: Institute de Estadística de la UNESCO. https://virtualeduca.org/documentos/ce ntrodocumentacion/2013/219369s.pdf

Uribe, A. (2015). Diseño de una propuesta curricular para docentes de la básica primaria, enfocada a la integración de las Tecnologías de la Información y las Comunicaciones (TIC) en el modelo Escuela Nueva - Escuela Activa, en la Institución Educativa Rural (IER) Santa Rosa. Medellín. 